\title{
Impact of bendiocarb $80 \%$ WP indoor residual spraying on insecticide resistance status of Anopheles arabiensis
}

\author{
Alemayehu Abate ${ }^{1,3,}{ }^{*}$ Emana Getu ${ }^{1}$, Melaku Wale ${ }^{2}$, Mamuye Hadis ${ }^{3}$, \\ Wubegzier Mekonen ${ }^{4}$ \\ ${ }^{1}$ Department of Zoological Sciences, Addis Ababa University, Ethiopia \\ ${ }^{2}$ Biology Department, Bahir Dar University, Ethiopia \\ ${ }^{3}$ Ethipian Public Health Institute, Addis Ababa, Ethiopia \\ ${ }^{4}$ School of Public Health, Addis Ababa University, Ethiopia
}

\begin{abstract}
Long term and wide use of indoor residual sprays augments the selection of insecticide resistance genes. The development of insecticide resistance in an insect population depends up on the volume and frequency of sprays against them and the inherent characteristics of the insect species. However, despite its use for decades, the effect of residual sprays on the status of insecticide resistance of malaria vectors is unknown in Ethiopia. The objective of this study was to assess the effect of bendiocarb $80 \%$ WP indoor residual spraying on insecticide resistance status of $A n$. arabiensis in Bahir Dar Zuria District, Northwest Ethiopia. Susceptibility of An. arabiensis was examined against different insecticides using 2-3-day old female mosquitoes following WHO insecticide susceptibility test procedures. Test results were calculated according to WHO bioassay test protocol. Chi-square test was used to determine the significance level of differences between years and study sites. Susceptibility of An. arabiensis to fenitrothion, pirimiphosmethyl and propoxur was $100 \%$ in both study villages for three successive years. Resistance to bendiocarb was suspected after two years (2015) in Andassa, but not in Tikurit. Mortality and knock down due to DDT significantly increased from 2013 to 2015 at Tikurit (knock down: $\chi^{2}=117.9, P<.0001$ and mortality: $\chi^{2}=66.3, P<.0001$; due to deltamethrin, knock down: $\chi^{2}=7.3, P=.004$ and mortality: $\left.\chi^{2}=37.8, P<.0001\right)$. The same was true at Andassa (due to DDT, knock down: $\chi^{2}=198.7, P<.0001$ and mortality: $\chi^{2}=$ 82.9, $P<.0001$; due to deltamethrin, knock down: $\chi^{2}=26.1, P<.0001$ and mortality: $\left.\chi^{2}=48.2, P<.0001\right)$. Bendiocarb was effective against the An. arabiensis for two years under bendiocarb IRS operation so that alternative insecticides with different mode of action should be replaced every two to three years to prolong its efficacy.
\end{abstract}

Keywords: Bendiocarb, Ethiopia, Insecticide resistance, Indoor residual spraying DOI: https://dx.doi.org/10.4314/ejst.v13i3.4

\footnotetext{
* Corresponding author: abate alemayehu@yahoo.com

(C)This is an Open Access article distributed under the terms of the Creative Commons Attribution License (http://creativecommons.org/licenses/CC BY4.0)
} 


\section{INTRODUCTION}

Insecticide resistance is a natural and heritable characteristic in an insect population that could be manifested by the failure of an insecticide product to provide the intended level of control when used as recommended for a particular species at a particular time and place. The magnitude of resistance development in an insect population depends up on the volume and frequency of the application of the insecticides used against them and the inherent characteristics of the insect species involved (Pates and Curtis, 2005; Brooke and Koekemoer, 2010). Studies indicated that a long-term wide application of indoor residual spraying (IRS) and use of long-lasting insecticide treated mosquito nets (LLINs) augments the selection of insecticide resistance gene frequencies (Stump et al., 2004; Reimer et al., 2005; Padonu et al., 2012).

Ethiopia has a long history of using IRS for its malaria vector control program and IRS remains to be one of the core interventions to control malaria vector. In most malarious parts of the country, a one-blanket round of IRS has been implemented for decades. There are also some areas where two rounds of IRS have been implemented mainly when epidemics occur. DDT has been the choice of insecticide for the country's IRS operations for decades and was replaced by deltamethrin in 2007. Malathion was also used to a limited extent over 10 years in some areas where high DDT resistance was detected (FDREMOH, 2012). Deltamethrin was also replaced by bendiocarb in 2010 due to the development of deltamethrin and other pyrethroid insecticide resistance in populations of the main malaria vector and by considering the impact of LLINs on resistance development.

Insecticide resistance is reversible and manageable. It could be the result of repeated application of the same insecticide, use of similar insecticides for agricultural pests or use of insecticides of different groups having similar mode of action. Therefore, the use of two or more insecticides having different modes of action alternatively within a given period could delay the development of insecticide resistance at a significant level. The removal of its selection pressure would help to restore susceptibility of vector population that was resistant to the insecticide under consideration (IRAC, 2011). Therefore, knowing the time required to delay or restore resistance to susceptibility is vital to use the available limited insecticides recommended for vector control. Different levels of insecticide resistance of $A n$. arabiensis have been reported against a range of insecticides of different classes, i.e., DDT, malathion, permethrin, deltamethrin, lambdacyhalothrin and bendiocarb (Meshesha Balkew et al., 2003; Alemayehu Abate and 
Mamuye Hadis, 2011; Dilnesaw Yehwalaw et al., 2011). The vector developed resistance not only to insecticides that had been used either for IRS or LLINs, but also to insecticides that had never been used before, which would be the outcome of cross-resistance. Therefore, if insecticide resistance is not managed, the success achieved in the country so far by means of IRS and LLIN to control malaria would be at risk. Thus, this study was conducted to estimate the effect of bendiocarb $80 \%$ WP indoor residual spraying on insecticide resistance status of An. arabiensis within three years.

\section{MATERIALS AND METHODS}

\section{Study site and study design}

A comparative study was carried out at Yegoma Huletu kebele at three

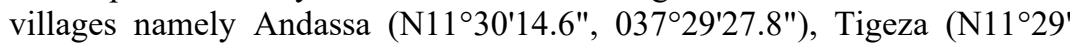

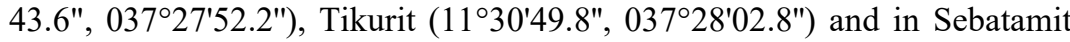
kebele at one village called Tikurit- Dewel (N11'31'14.2", E037²3'52.3"). The study villages were categorized into two study groups, Andassa and Tigeza in one group, named as Andassa; and Tikurit and Tikurit-Dewel in the second group, and named as Tikurit. Grouping was made based on their proximity to each other, while other factors were considered similar. Susceptibility status of An. arabiensis to different insecticides was assessed for three consecutive years. Andassa received three round sprays, i.e., one spray in 2013, 2014 and 2015 each, while Tikurit was sprayed only in 2015.

\section{WHO insecticide susceptibility testing}

Groups of 2-3-days old females reared under field insectarium were selected using WHO tubes and exposed to papers treated with different insecticides including DDT $(4 \%)$, deltamethrin $(0.05 \%)$, malathion $(5 \%)$, bendiocarb $(0.1 \%)$, propoxur $(1 \%)$, fenitrothion $(1 \%)$ and pirimiphosmethyl $(1 \%)$. Insecticide treated papers were purchased from the WHO reference Centre at University Sains, Penang, Malaysia. A minimum of 100 mosquitoes/insecticide type were exposed for a standard one-hour susceptibility test and mortality was recorded after 24 hours holding period.

\section{Detection of knockdown resistance}

Two separate PCR reactions were run, one to detect $k d r-e$ and the other $k d r$ $w$ at a time (Figures $1 \& 2$ ). Extracted DNA was stored at $-80{ }^{\circ} \mathrm{C}$ and used 
for kdr PCR amplification. The master mix formulation for detecting the DNA changes responsible for $\mathrm{kdr}$ in An. arabiensis was consisted of Acustar II (consisted of Taq polymerase 5 units/ $\mu$, dATP, dCTP, dGTP, dTTP, $\mathrm{Mgcl}_{2}$, 5xbuffer) molecular grade water, primers and DNA templates. East and west resistance alleles were distinguished using east or west primers along with the WT (Table 1). The final volume of the master mix for kdr amplification was $25 \mu \mathrm{l}$ (Table 2). Thermal cycling for kdr PCR reaction was programmed at Bio-Rad PCR machine for 30 cycles with an initial denaturing step at $95{ }^{\circ} \mathrm{C}$ for 30 seconds, annealing at $57{ }^{\circ} \mathrm{C}$ for 30 seconds (for kdr east) or at $59{ }^{\circ} \mathrm{C}$ for 30 seconds (for kdr west), extension at $72{ }^{\circ} \mathrm{C}$ for 30 seconds and at $72{ }^{\circ} \mathrm{C}$ for 5 minutes.

Table 1. Primers and their sequences used to identify DNA changes in An. arabiensis

\begin{tabular}{ll}
\hline Primer name & Primer sequence \\
\hline IPCF-F & GATAATGTG GATAGATTCCCC GACCAT G \\
AltRev- R & TGC CGT TGG TGC AGA CAA GGA TG \\
EAST: WT-R & GGTCCATGTTAATTTGCATTACTTACGATA \\
East: F & CTTGGCCACTGTAGTGATAGGAAAaTC \\
IPCF-F & GATAATGTG GATAGATTCCCC GACCAT G \\
AltRev- R & TGCCGTTGGTGCAGACAAGGATG \\
WEST: WT-R & GGTCCATGTTAATTTGCATTACTTACGAaTA \\
West $-\mathrm{F}$ & CTT GGC CAC TGT AGT GAT AGG AAA TgTT \\
\hline
\end{tabular}

\section{Visualization of amplified DNA on a $2 \%$ agarose gel}

The PCR products were separated by electrophoresis on $2 \%$ agarose TBE gels. A $1.4 \mathrm{~g}$ of agarose gel was dissolved in $70 \mathrm{ml}$ of buffer and stained by $7 \mu \mathrm{l}$ of gel red. The solution was then microwaved for 2 minutes and cooled by running cold tap water on the outside of heating flask poured into an electrophoresis tray where 14 or 20 well comb was inserted. The comb was removed after the agarose gel was completely cooled. Three micro liters of the PCR product was loaded into each well and run at $70-75$ volts for $2 \frac{1}{2}$ hours. A negative control was also loaded in a well per assay. The amplicons were visualized with an ultraviolet trans-illumination gel documentation system (Alpha Imager 2200, San Leandro, California, USA). The predicted DNA bands on the gel were compared to a one kb reference DNA ladder (Figures $1 \& 2$ ). 


\section{Data analyses}

Insecticide susceptibility test results were calculated according to WHO (2013) bioassay test protocol. Chi-square test was used to determine level of significance of differences in knockdown rates and mortalities within and between the study sites and years. Line graphs were used to show susceptibility status of An. arabiensis to different insecticides against time. Gel photographs show the bands of resistance alleles in DDT and deltamethrin resistant An. arabiensis after separating them on 2\% agarose gel.

Table 2. PCR components for kdr detection in An. Arabiensis

\begin{tabular}{lll}
\hline Components & Concentration & Volume/25 $\boldsymbol{\mu l}$ rxn \\
\hline Accustar II & & $12.5 \mu \mathrm{l}$ \\
IPCF-F & $2.5 \mu \mathrm{M}$ & $2 \mu \mathrm{l}$ \\
EAST AltRev-R & $2.5 \mu \mathrm{M}$ & $2 \mu 1$ \\
EAST: WT-R & $5 \mu \mathrm{M}$ & $2 \mu \mathrm{l}$ \\
EAST: WT-F & $2.5 \mu \mathrm{M}$ & $2 \mu 1$ \\
$\mathrm{H}_{2} \mathrm{O}$ & Biological Grade & $2.5 \mu \mathrm{l}$ \\
DNA templates & Mosquito extract & $2 \mu \mathrm{l}$ \\
Total volume/well & & $25 \mu \mathrm{l}$ \\
Accustar II & & $12.5 \mu \mathrm{M}$ \\
IPCF-F & $2.5 \mu \mathrm{M}$ & $1 \mu 1$ \\
West AltRev-R & $2.5 \mu \mathrm{M}$ & $1 \mu \mathrm{l}$ \\
West: WT-R & $25 \mu \mathrm{M}$ & $1 \mu 1$ \\
West: WT-F & $8.8 \mu \mathrm{M}$ & $3 \mu \mathrm{l}$ \\
$\mathrm{H}_{2} \mathrm{O}$ & Biological Grade & $4.5 \mu \mathrm{l}$ \\
DNA templates & Mosquito extract & $2 \mu \mathrm{l}$ \\
Total volume/well & & $25 \mu \mathrm{l}$ \\
\hline
\end{tabular}

\section{RESULTS}

\section{Effect of IRS on insecticide resistance status of $\mathbf{A n}$. arabiensis}

Susceptibility of An. arabiensis to fenitrothion, pirimiphosmethyl and propoxur was $100 \%$ in both study villages for three successive years. The same was true for bendiocarb in Tikurit. However, in Andassa, resistance to bendiocarb was suspected after three spraying cycles in three years' time because susceptibility of the vector to this insecticide was $<98 \%$. However, there was no significant difference in susceptibility of the vector to 
bendiocarb within the same study site between years $\left(\chi^{2}=0.25, P=.8825\right.$ in Andassa; $\chi^{2}=0.02, P=.99$ in Tikurit) (Table 3).

Susceptibility status of An. arabiensis to malathion also steadily reversed in both villages with time (Table 4). While susceptibility of An. arabiensis was below $98 \%$ in Andassa, the vector was $100 \%$ susceptible to malathion in Tikurit in 2015. Reversion of resistance to susceptibility was significant within and between study villages (Tables 3). Mortality due to malathion significantly increased from 2013 to 2015 (Andassa: $\chi^{2}=8.04, P=.02$; Tikurit: $\chi^{2}=8.04, P=.0018$ ).

Table 3. Change in susceptibility status of An. arabiensis exposed to differ insecticides in Andassa \& Tikurit (2013-2015).

\begin{tabular}{|c|c|c|c|c|c|c|}
\hline \multirow[b]{2}{*}{ Study site } & \multirow[b]{2}{*}{ Effect } & \multicolumn{3}{|c|}{ Year } & \multirow[b]{2}{*}{$\chi^{2}$} & \multirow[b]{2}{*}{$P$ value } \\
\hline & & 2013 & 2014 & 2015 & & \\
\hline & & \multicolumn{3}{|l|}{ DDT } & \multirow{3}{*}{4.54} & \multirow{3}{*}{1.04} \\
\hline Andassa & Knock down & 15 & 21 & 20 & & \\
\hline Tikurit & Knockdown & 23 & 33 & 63 & & \\
\hline Andassa & Mortality & 33 & 42 & 79 & \multirow[t]{2}{*}{1.89} & \multirow[t]{2}{*}{0.39} \\
\hline Tikurit & Mortality & 38 & 52 & 78 & & \\
\hline \multicolumn{7}{|c|}{ Deltamethrin } \\
\hline Andassa & Knock down & 55 & 78 & 90 & \multirow[t]{2}{*}{1.45} & \multirow[t]{2}{*}{0.48} \\
\hline Tikurit & Knockdown & 73 & 81 & 100 & & \\
\hline Andassa & Mortality & 43 & 67 & 78 & \multirow[t]{2}{*}{0.2} & \multirow[t]{2}{*}{0.9} \\
\hline Tikurit & Mortality & 46 & 70 & 90 & & \\
\hline & & \multicolumn{3}{|c|}{ Malathion } & \multirow{3}{*}{15201111} & \multirow{3}{*}{0} \\
\hline Andassa & Mortality & 78 & 84 & 92 & & \\
\hline Tikurit & Mortality & 24 & 71 & 100 & & \\
\hline & & \multicolumn{3}{|c|}{ Bendiocarb } & \multirow{3}{*}{0.7493} & \multirow{3}{*}{0.96} \\
\hline Andassa & Mortality & 100 & 100 & 95 & & \\
\hline Tikurit & Mortality & 99 & 100 & 99 & & \\
\hline
\end{tabular}

An. arabiensis was resistant to DDT and deltamethrin in both study villages throughout the study period (Tables $3 \& 4$ ). However, the level of susceptibility of the vector to these insecticides improved progressively and significantly with time. Mortality and knock down effect due to DDT significantly increased from 2013 to 2015 (knock down (kd): $\chi^{2}=117.87$, $P<.0001$ and mortality: $\left.\chi^{2}=66.3, P<.0001\right)$ in Tikurit. The same was true for deltamethrin (kd: $\chi^{2}=7.3, P=.004$ and mortality: $\left.\chi^{2}=37.8, P<.0001\right)$. Similarly, mortality and knock down effect due to DDT significantly increased from 2013 to 2015 (kd: $\chi^{2}=198.7, P<.0001$ and mortality: $\chi^{2}=$ $82.9, P<.0001)$ in Andassa. The trend was also similar for deltamethrin $(\mathrm{kd}:$ 
$\chi^{2}=26.1, P<.0001$ and mortality: $\left.\chi^{2}=48.2, P<.0001\right)$. The difference in the reversion of resistance to susceptibility of the vector to DDT and deltamethrin between the study villages was not significant $(P>.05)$ (Table $3)$.

Table 4. Susceptibility status of An. arabiensis to DDT, deltamethrin, malathion, bendiocarb, propoxur \& pirimiphosmethyl insecticides at Andassa and Tikurit across years (2013-2015).

\begin{tabular}{llllllll}
\hline & \multicolumn{3}{c}{ Andassa } & & \multicolumn{3}{c}{ Tikurit } \\
\cline { 2 - 3 } \cline { 7 - 8 } Insecticides & $\mathbf{2 0 1 3}$ & $\mathbf{2 0 1 4}$ & $\mathbf{2 0 1 5}$ & & $\mathbf{2 0 1 3}$ & $\mathbf{2 0 1 4}$ & $\mathbf{2 0 1 5}$ \\
\hline DDT (4\%) & 33 & 42 & 79 & & 38 & 52 & 78 \\
Deltamethrin (0.05\%) & 43 & 67 & 78 & & 46 & 70 & 90 \\
Malathion (5\%) & 78 & 84 & 92 & & 24 & 71 & 100 \\
Bendiocarb (0.1\%) & 100 & 100 & 95 & & 99 & 100 & 100 \\
Propoxur (1\%) & 100 & 100 & 100 & & 100 & 100 & 100 \\
Pirimiphosmethyl (1\%) & 100 & 100 & 100 & & 100 & 100 & 100 \\
Fenitrothion (1\%) & 100 & 100 & 100 & & 100 & 100 & 100 \\
\hline
\end{tabular}

Detection of DDT and deltamethrin kdr mutation in An. arabiensis

Figures 1 and 2 show the presence or absence of kdr alleles in $A n$. arabiensis and the changes in kdr mutations with time. The East African kdr $(k d r-e)$ was detected in subsample of An. arabiensis screened for the presence of kdr mutations, while the West African $\mathrm{kdr}(k d r-w)$ was absent.

Gel photographs showed bands of $314 \mathrm{bp}$ signifying that PCR reactions were successful. Bands of 156 indicated that the vector had homozygous resistant allele. Other bands showed heterozygous resistance alleles of the vector. The change from homozygous to heterozygous kdr was higher for DDT than deltamethrin within three years. 


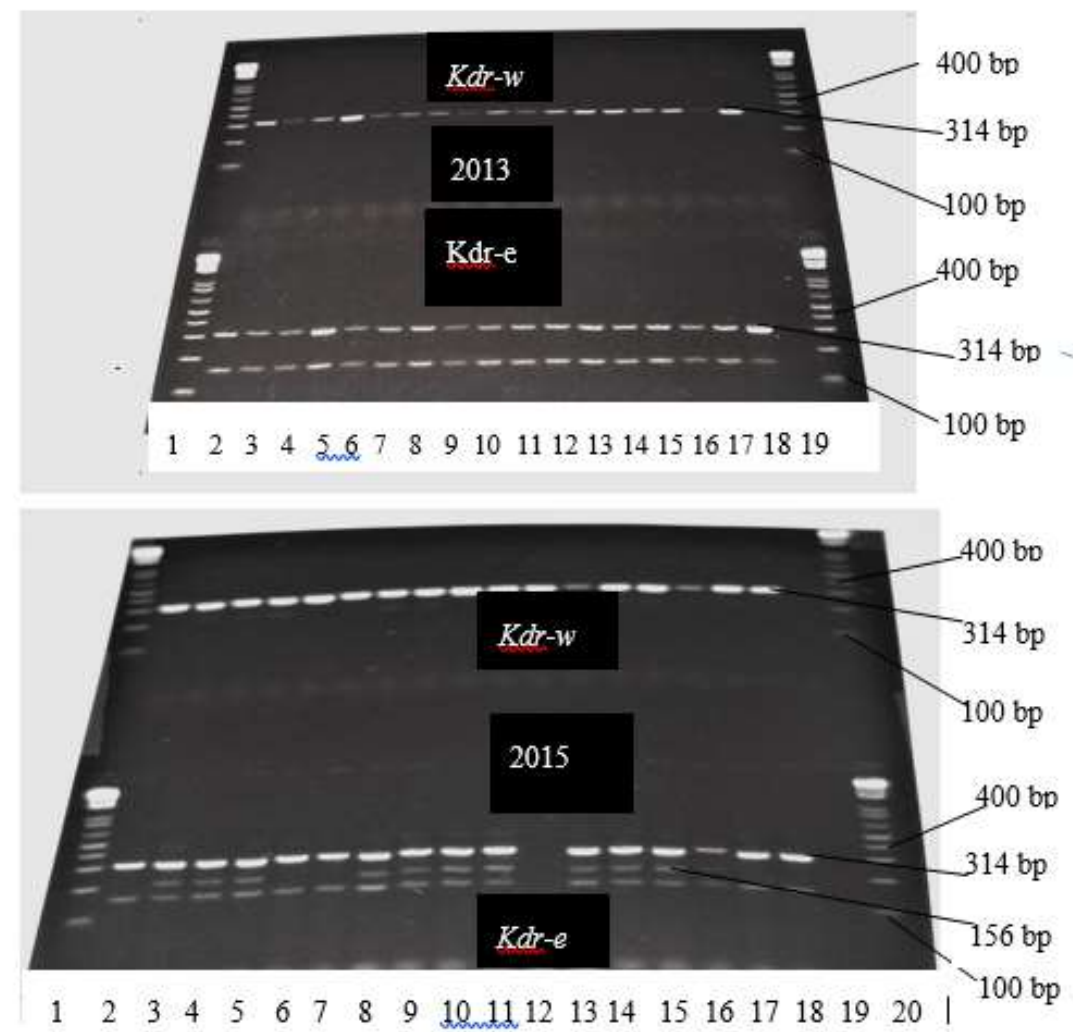

Figure 1. Gel photographs showing bands of DDT resistant kdr alleles in An. arabiensis after separation on $2 \%$ agarose gel: lane $1 \& 201 \mathrm{~kb}$ ladder, 19 negative control (2013) 2-18 homozygous resistant (2015) 2, 6, 7, 16-18 homozygous resistant \& 3-5, 8-11, 13-15 heterozygous resistant, 12 not amplified, no $k d r-w$ detected both in 2013 \& 2015. 


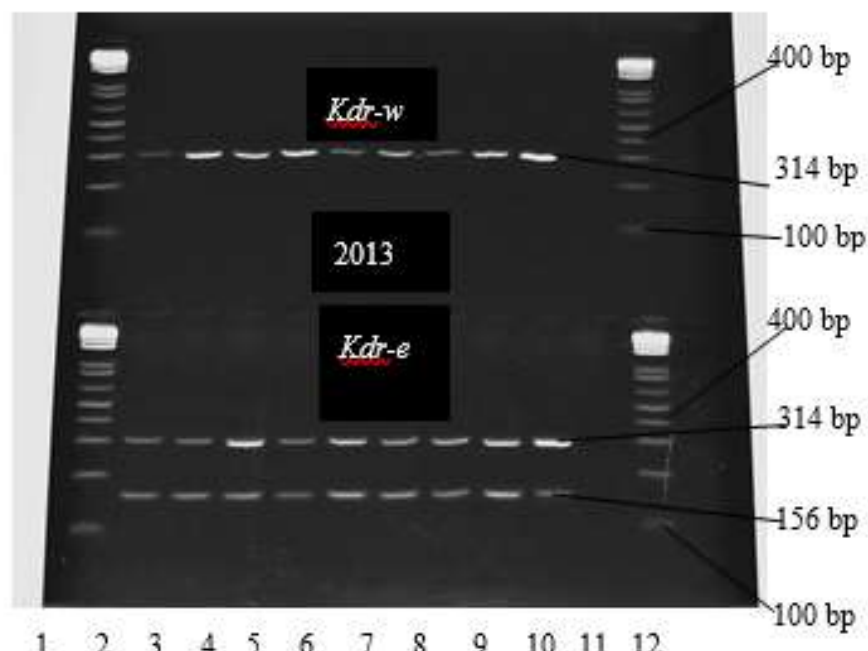

$\begin{array}{llllllllllll}1 & 2 & 3 & 4 & 5 & 6 & 7 & 8 & 9 & 10 & 11 & 12\end{array}$

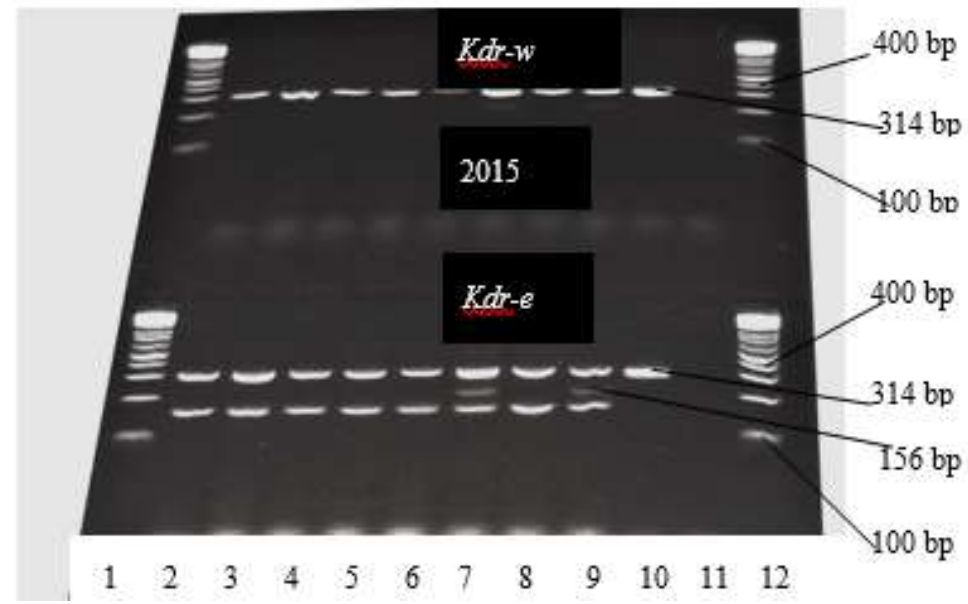

Figure 2. Gel photographs showing bands of deltamethrin resistant kdr alleles in An. arabiensis after separation on 2\% agarose gel: lane 1 \& $121 \mathrm{~kb}$ ladder, 11 negative control (2013) 2-10 homozygous resistant (2015) 2-6 \& 8 homozygous resistant, $7 \& 9$ heterozygous resistant, 10 not amplified, no $\mathrm{kdr}-\mathrm{w}$ detected both in $2013 \& 2015$. 


\section{DISCUSSION}

Genes conferring resistance to insecticides are natural and heritable in an insect population. Insecticide resistance in a given vector population is the selection of these heritable characteristics. The selection process would be slow if the resistance genes are rare or present at low rate in vector population and the effect of selection pressure for resistance lasts shorter (IRAC, 2011). The operational life of an insecticide could vary with the amount and the frequency of its application (Koella et al., 2012). The significant increase in insecticide resistance in malaria vector populations globally especially in Africa is due to the large scale and extensive application of insecticides in the form of IRS and LLINs for vector control (WHO, 2012).

Resistance to DDT (dichlorodiphenyltrichloroethane) was first reported in Aedes mosquito a year after the introduction of this insecticide for mosquito control in 1946 (Brown, 1986). DDT resistance was also detected in malaria vectors developed by the end of 1960s following its widespread application during the era of malaria eradication started in 1955 (Hemingway and Ranson, 2000; Kelly-Hope et al., 2008). DDT had been used for more than four decades in Ethiopia and populations of the main malaria vector in the country are resistant to DDT throughout the country. In the present study, resistance to bendiocarb was suspected after three spraying cycles in three years' time implemented through one round spray/year. Compared with DDT, the effective residual life of bendiocarb is shorter. This could be attributed to the differences in their mode of action and the inherit characteristics of the vector species involved. The differences observed in the residual life of bendiocarb between Tikurit and Andassa could be justified by the difference in the frequency of bendiocarb IRS spraying cycles, i.e., Tikurit received one spraying cycle while Andassa received three, indicating the need to alternate bendiocarb by other insecticide having different mode of action every 2-3 years.

Insecticide resistance is reversible when its selection pressure is removed (IRAC, 2011). Published data on the time required to revert resistance to susceptibility are limited and difficult to discuss the present data in relation to other studies. The present study demonstrated that resistance reverted to susceptibility every year progressively. Susceptibility of An. arabiensis to malathion was reverted from $24 \%$ to $100 \%$ in Tikurit in three years while the same vector was found to be resistant in Andassa. The possible reason for the differences observed in the rate of reversion in malathion resistance in the two villages might be due to the selection pressure exerted by 
bendiocarb IRS in Andassa since both insecticides have similar mode of action. The trend in the reversion of DDT and deltamethrin resistance to susceptibility was similar in both study villages justifying that the impact of bendiocarb IRS application or other selection pressure for these insecticides is low or absent.

Two mutations, which are known as $k d r-e$ and $k d r$-w, occurring at the same locus in the voltage-gated sodium channel gene are known to confer knockdown resistance $(\mathrm{kdr})$ to pyrethroids and DDT. Kdr-e involves a leucine-serine substitution while $k d r$ - $w$ involves a leucine-phenylalanine substitution. Several studies conducted before indicate that $k d r$-w predominated West Africa (Fanello et al., 2000; Diabate et al., 2002; Awolola et al., 2005; Dabire et al., 2009) and kdr-e East Africa (Ranson et al., 2000; Verhaeghen et al., 2006). However, $k d r-e$ alleles have been spread out in populations of An. arabiensis in West African countries including Burkina Faso and Benin (Djegbe et al., 2011; Badolo et al., 2012) recently. Similarly, $k d r-w$ has been distributed in vector populations in Uganda (Verhaeghen et al., 2006) and Ethiopia (Dilnesaw Yehwalaw et al., 2010; Meshesha Balkew et al, 2010). In this study, only kdr-e was detected and may be attributable to DDT and deltamethrin resistance in $A n$. arabiensis. The results of the present study agree with previous studies reported from different African countries (Djegbe et al., 2011; Badolo et al., 2012) where both $k d r-w$ and $k d r-e$ were found within the same country. However, in Ethiopia, Dilnesaw Yehwalaw et al. (2010) and Meshesha Balkew et al. (2010) reported $k d r-w$, which is inconsistent with the present results. The differences observed between the previous and present studies in Ethiopia might be due to the recent inflow of $k d r-e$ genes in $A n$. arabiensis populations. Therefore, the differences observed between the previous and the present study in Ethiopia needs to be verified.

\section{CONCLUSION}

The present study confirmed that bendiocarb would be effective against $A n$. arabiensis for three years under bendiocarb IRS operation. Therefore, alternative insecticides with different mode of action should be used to replace every two to three years to delay the development of resistance to bendiocarb. The present study was conducted in two study sites. Therefore, a longitudinal study should be carried out in more representative study sites in the country to determine the time to either rotate insecticides in IRS operations to delay IR development or restore susceptibility of the vector to the insecticides that the vector already developed resistance. 


\section{ACKNOWLEDGMENTS}

Ethiopian Public Health Institute is acknowledged for sponsoring the study.

\section{REFERENCES}

Alemayehu Abate and Mamuye Hadis (2011). Susceptibility of Anopheles gambiae s.l to DDT, malathion, permethrin and deltamethrin in Ethiopia. Tropical Medicine and International Health 6: 486-91.

Awolola, T.S., Oyewole, I.O., Amajoh, C.N., Idowu, E.T., Ajayi, M.B., Oduola, A., Manafa, O.U., Ibrahim, K., Koekemoer, L.L and Coetzee, M. (2005). Distribution of the molecular forms of Anopheles gambiae and pyrethroid knock down resistance gene in Nigeria. Acta Tropica 95: 204-209.

Badolo, A., Traore, A., Jones, C.M., Sanou, A., Flood, L.M, Guelbeogo, W.M., Ranson, H and Sagnon, N.F. (2012). Three years of insecticide resistance monitoring in Anopheles gambiae in Burkina Faso: resistance on the rise? Malaria Journal 11: 232.

Brooke, B.D and Koekemoer, L.L. (2010). Major effect genes or loose confederations? The development of insecticide resistance in the malaria vector Anopheles gambiae. Parasites \& Vectors 3: 74.

Brown, A.W.A. (1986). Insecticide resistance in mosquitoes: a pragmatic review. Journal of American Mosquito Control Association 2: 123 -140.

Dabire, K.R., Diabate, A., Namountougou, M., Toé, K.H., Ouari, A., Kengne, P., Bass, C and Baldet, T. (2009). Distribution of pyrethroid and DDT resistance and the L1014F kdr mutation in Anopheles gambiae s.l from Burkina Faso (West Africa). Transactions of the Royal Society of Tropical Medicine \& Hygiene 103: 1113-1120.

Diabate, A., Baldet, T., Chandre, F., Akogbéto, M., Guiguemde, T.R., Akogbeto, M., Robert, T., Guiguemde, F.D, Brengues, C., Guillet, P., Hemingway, J., Small, G.J and Hougard, J.M. (2002). The role of agricultural use of insecticides in resistance to pyrethroids in Anopheles gambiae s.l in Burkina Faso. American Journal of Tropical Medicine and Hygiene 67: 617-622.

Dilnesaw Yehwalaw, Bortel, W.V., Denis, L., Cosemans, M., Duchateau, L and Speybroeck, N. (2010). First Evidence of High Knockdown Resistance Frequency in Anopheles arabiensis (Diptera: Culicidae) from Ethiopia. American Journal of Tropical Medicine and Hygiene 83(1): 122-125.

Dilnesaw Yewhalaw, Fentahun Wassie, Steurbaut, W., Spanoghe, P., Bortel, Spanoghe, P., Bortel, W.V., Denis, L., Dejene A Tesema, Yehenew Getachew, Marc Coosemans, M., Luc Duchateau, L and Speybroeck, N. (2011). Multiple insecticide resistance: an impediment to insecticide-based malaria vector control program. PLoS ONE 6(1): e16066 doi:10.1371/journal.pone.001606. Accessed on $11 / 25 / 2016$

Djegbe, I., Boussari, O., Sidick, A., Martin, T., Ranson, H., Martin, T., Ranson, H., Chandre, F., Akogbéto, M and Vincent Corbel, V. (2011). Dynamics of insecticide resistance in malaria vectors in Benin: First evidence of the presence 
of L1014S kdr mutation in Anopheles gambiae from West Africa. Malaria Journal 10: 261.

Fanello, C., Akogbéto, M and della Torre, A. (2000). Distribution of the knock down resistance gene (kdr) in Anopheles gambiae s.l from Benin. Transactions of the Royal Society of Tropical Medicine and Hygiene 94: 13

FDREMOH (2012). National malaria guideline. Third edition. The Federal Democratic Republic of Ethiopia, Ministry of Health. Addis Ababa, Ethiopia.

Hemingway, J and Ranson, H. (2000). Insecticide resistance in insect vectors of human disease. Annual Review of Entomology 45: 371-391.

IRAC (2011). Prevention and management of insecticide resistance in vectors and pests of health importance, $2^{\text {nd }}$ edition. A manual produced by: Insecticide resistance action committee. 72 pages.

Kelly-Hope, L., Ranson, H and Hemingway, J. (2008). Lessons from the past: managing insecticide resistance in malaria control and eradication programs. The Lancet Infection Diseases 8: 387-389.

Koella, J.C., Saddler, A., Thomas, P.S and Karacsow, T.P.S. (2012). Blocking the evolution of insecticide resistant malaria vectors with a microsporidian. Evolutionary Applications 5(3): 283-292.

Meshesha Balkew, Ibrahim, M., Koekemoer, L.L., Brooke, B.D., Howard Engers, H., Abraham Aseffa, Teshome Gebre-Michael and Elhassen, I. (2010). Insecticide resistance in Anopheles arabiensis (Diptera: Culicidae) from villages in central, northern and southwest Ethiopia and detection of kdr mutation. Parasites \& Vectors 3: 40.

Meshesha Balkew, Teshome Gebre-Michael and Asrat Hailu. (2003). Insecticide susceptibility of Anopheles arabiensis in two agro-development localities in eastern Ethiopia. Parasitologia 45: 1-3.

Padonu, G.G., Gbedjissi, G., Yadouleton, A., Azondekon, R., Razack, O., Oussou, O., Gnanguenon, V., Rock, A.R., Sezonlin, M and Akogbeto, A. (2012). Decreased proportions of indoor feeding and endophily in Anopheles gambiae s.l populations following the indoor residual spraying and insecticide-treated net interventions in Benin (West Africa). Parasites \& Vectors 5: 262.

Pates, H and Curtis, C. (2005). Mosquito behavior \& vector control. Annual Review of Entomology 50: 53-70.

Ranson, H., Jensen, B., Vulule, J.M., Wang, X., Hemingway, J., Collins, F.H. (2000). Identification of a point mutation in the voltage-gated sodium channel gene of Kenyan Anopheles gambiae associated with resistance to DDT and pyrethroids. Insect Molecular Biology 9: 491-497.

Reimer, L.J., Tripet, F., Slotman, M.., Spielman, A., Fondjo, E and Lanzaro, G.C. (2005). The Royal Entomological Society 683 Blackwell Publishing, Ltd. An unusual distribution of the kdr gene among populations of Anopheles gambiae on the island of Bioko, Equatorial Guinea. Insect Molecular Biology 14(6): 683688.

Stump, A.D., Atieli, F.K., Vulule, J.M and Besansky, N.J. (2004). Dynamics of the pyrethroids knockdown resistance in Western Kenyan populations of Anopheles gambiae in response to insecticide-treated bed net trials. American Journal of Tropical Medicine and Hygiene 70: 591-596. 
Verhaeghen, K., Van Bortel, W., Roelants, P., Backeljau, T and Coosemans, M. (2006). Detection of the East and West African kdr mutation in Anopheles gambiae and Anopheles arabiensis from Uganda using a new assay based on FRET/Melt Curve analysis. Malaria Journal 5: 16.

WHO (2012). Global plan for insecticide resistance management in malaria vectors. ISBN 9789241564472.

WHO (2013). Test procedures for insecticide resistance monitoring in malaria vector mosquitoes. World Health Organization. Genève. 